\title{
IncRNA C22orf32-1 contributes to the tumorigenesis of nasopharyngeal carcinoma
}

\author{
GUO-HUI NIE ${ }^{1 *}$, ZHAO LI $^{2,3^{*}}$, HONG-FANG DUAN ${ }^{4}$, LIANG LUO ${ }^{2,3}$, HONG-YI HU ${ }^{2}$, \\ WEI-QIANG YANG ${ }^{2}$, LI-PING NIE ${ }^{5}$, RU-FEI ZHU ${ }^{2}$, XIAO-FAN CHEN $^{6}$ and WEI ZHANG ${ }^{6}$ \\ ${ }^{1}$ Department of Otolaryngology, Shenzhen Second People's Hospital, Shenzhen, Guangdong 518035; \\ ${ }^{2}$ Department of Otolaryngology, Peking University Shenzhen Hospital, Shenzhen, Guangdong 518036; \\ ${ }^{3}$ Guangzhou Medical University, Guangzhou, Guangdong 510000; ${ }^{4}$ Department of Otolaryngology, \\ Shenzhen Children's Hospital, Shenzhen, Guangdong 518038; ${ }^{5}$ Department of Clinical Laboratory, \\ Peking University Shenzhen Hospital; ${ }^{6}$ Biomedical Research Institute, Shenzhen Peking University-
}

The Hong Kong University of Science and Technology Medical Center, Shenzhen, Guangdong 518036, P.R. China

Received March 13, 2016; Accepted February 13, 2017

DOI: $10.3892 / 01.2017 .6021$

\begin{abstract}
The mechanism of nasopharyngeal carcinoma (NPC) remains unclear. The present study investigated the abnormal expression of long non-coding (lnc)RNAs in NPC tissues and one NPC cell line to identify the involvement of lncRNAs in the tumorigenesis of NPC. Using a quantitative reverse transcription polymerase chain reaction (RT-qPCR), the expression of lncRNA C22orf32-1 in NPC tissues and an NPC cell line was verified. The effects of IncRNA C22orf32-1 on NPC cells were investigated with a cell proliferation assay, cell scratch assay, Transwell assay and a cell apoptosis assay. The expression levels of lncRNA C22orf32-1 in NPC tissues and an NPC cell line were upregulated. IncRNA C22orf32-1 promoted the proliferation, migration and invasion of NPC cells, and reduced the apoptosis of NPC cells. The data demonstrated that IncRNA C22orf32-1 may facilitate the tumorigenesis of NPC, and may be used for the early diagnosis and treatment of NPC.
\end{abstract}

Correspondence to: Dr Guo-Hui Nie, Department of Otolaryngology, Shenzhen Second People's Hospital, 3002 Sungang West Road, Shenzhen, Guangdong 518035, P.R. China

E-mail:nghui@21cn.com

Dr Wei Zhang, Biomedical Research Institute, Shenzhen Peking University-The Hong Kong University of Science and Technology Medical Center, 1120 Lotus Road, Shenzhen, Guangdong 518036, P.R. China

E-mail: zhangweispace@yeah.net

*Contributed equally

Key words: nasopharyngeal carcinoma, lncRNA C22orf32-1, oncogene, tumorigenesis, biomarker

\section{Introduction}

Nasopharyngeal carcinoma (NPC) is the most common type of malignancy of the neck and head in China $(1,2)$. Due to the anatomical location, the nasopharynx is difficult to access and the early symptoms of NPC are not distinctive. Therefore, NPC is usually diagnosed at the advanced stage $(3,4)$. At present, the main treatment of NPC is radiotherapy. Radiotherapy may inhibit the development of NPC, but it may seriously influence the quality of life of the patient due to the side effects (5-7). The regulatory mechanisms of NPC remain unclear. Therefore, research on the molecular mechanisms and genetic alterations involved in the progression of NPC is required, as this may help to find novel therapeutic and diagnostic methods (8-11).

Long non-coding (lnc)RNAs are non-protein-coding transcripts with $>200$ nucleotides (12). As the non-coding RNAs do not serve as the template for protein synthesis, they are considered superfluous in transcription. Multiple previous studies have demonstrated that lncRNAs take part in the development of numerous diseases, particularly via gene silencing, cell cycle regulating, chromatin remodeling and splicing regulating (13-15). The lncRNAs are categorized into oncogenes and tumor suppressor genes according to their different functions in variety of types of malignancy $(16,17)$. It was demonstrated that the dysfunctionally expressed lncRNAs were associated with the occurrence of tumors (18). Therefore, IncRNAs may be used for the early diagnosis of tumors and as novel targets for treatments $(19,20)$. lncRNA metastasis associated lung adenocarcinoma transcript 1 has already been used as a biomarker for the early diagnosis of non-small cell lung cancer, due to its specificity compared with the other serum-specific antigens (21).

lncRNA C22orf32-1 (OTTHUMG00000150697) was identified through gene expression profile analysis (22). The expression of IncRNA C22orf32-1 was significantly upregulated in primary NPC tissues compared with the normal nasopharyngeal epithelial tissues, and therefore may act as an 
independent biomarker for early diagnosis and provide a novel therapeutic target for NPC $(13,22,23)$. In the present study, the expression levels of lncRNA C22orf32-1 in 24 primary NPC tissues and 24 normal nasopharyngeal epithelial tissues were examined with one NPC and one normal nasopharyngeal epithelial cell line. The effect of lncRNA C22orf32-1 knockdown on NPC cell proliferation, migration, invasion and apoptosis was explored.

\section{Materials and methods}

Tissue specimens. A total of 48 samples were collected from Peking University Shenzhen Hospital (Shenzhen, China) from May 2013 to February 2015. Of these, 24 samples were NPC tissues from patients with primary NPC and others were normal nasopharyngeal epithelial from volunteers without cancer. Fresh samples of normal nasopharyngeal epithelium and NPC tissues were obtained from the Department of Head and Neck (Peking University Shenzhen Hospital, Shenzhen, China), and stored immediately in liquid nitrogen. The present study was approved by the Research Ethics Board at Peking University Shenzhen Hospital (Shenzhen, China). No NPC patients received radiotherapy or chemotherapy prior to the surgery. The clinical staging of all patients were determined using the 2010 Cancer Staging Standard published by the America Joint Committee (24). Details of the patient clinical information are summarized in Table I.

Cell culture. The normal human nasopharyngeal NP460 and NPC 6-10B cell lines were obtained from the Southern Medical University (Guangzhou, China) and Peking University Shenzhen Hospital (Shenzhen, China). Cells were grown in a humidified incubator with $5 \% \mathrm{CO}_{2}$ at $37^{\circ} \mathrm{C}$ and maintained in medium containing 88\% RPMI-1,640 medium (Gibco; Thermo Fisher Scientific, Inc., Waltham, MA, USA), 10\% fetal bovine serum (FBS; Gibco; Thermo Fisher Scientific, Inc.), $50 \mathrm{U} / \mathrm{ml}$ streptomycin and $50 \mathrm{U} / \mathrm{ml}$ penicillin (Gibco; Thermo Fisher Scientific, Inc.).

RNA extraction, complimentary (c)DNA synthesis and quantitative reverse transcription polymerase chain reaction (RT-qPCR). The RNA was extracted from samples and cell lines using the TRIzol reagent (Invitrogen; Thermo Fisher Scientific, Inc.) according to the protocol of the manufacturer. The purity of RNA was examined in the A260/A280 ratio using the SmartSpec Plus Spectrophotometer (Bio-Rad Laboratories, Inc., Hercules, USA). The cDNA was synthesized from RNA using a Reverse Transcription kit (Takara Biotechnology Co., Ltd., Dalian, China). The IncRNA C22orf32-1 expression levels in samples and cell lines were detected with the SYBR Green kit (Takara Biotechnology Co., Ltd.), and analyzed using the Roche Lightcycler 480 Real-Time PCR System (Roche Applied Science, Penzberg, Germany). The thermocycling conditions for RT-qPCR were: $94^{\circ} \mathrm{C}$ for $3 \mathrm{~min}$, then $94^{\circ} \mathrm{C}$ for $30 \mathrm{sec}, 55^{\circ} \mathrm{C}$ for $30 \mathrm{sec}$ and $72^{\circ} \mathrm{C}$ for $2 \mathrm{~min}$ for 40 cycles and finally $72^{\circ} \mathrm{C}$ for $10 \mathrm{~min}$. All samples were tested in triplicate. Glyceraldehyde 3-phosphate dehydrogenase (GAPDH) was set as the internal control to normalize the relative expression level of lncRNA C22orf32-1. The primer sequences were: Forward, 5'-AGC ACTTGGCCCTAAAGAGA -3'; reverse, 5'-AACATACTG
GCCCAAACAGC -3' for IncRNA C22orf32-1, and forward, 5'-GAGCACAGAGCCTCGCCTTT-3'; reverse, 5'-TCATCA TCCATGGTGAGCTGGC-3' for GAPDH. The expression levels of 1ncRNA C22orf32-1 and GAPDH in specimens were calculated using the $\Delta \Delta \mathrm{Cq}$ method $\left[\Delta \Delta \mathrm{Cq}=\left(\mathrm{meanCq}_{\mathrm{lncRNA}}\right.\right.$ C22orf32-1(NPC) -meanCq $_{\text {GAPDH (NPC) }}$-(meanCq IncRNA C22orf32-1(normal) $-\mathrm{m}$ eanC( $\left.\left.\mathrm{q}_{\mathrm{GAPDH}(\text { normal }}\right)\right]$. The relative RNA expression in cells was calculated using the $2^{-\Delta \Delta \mathrm{Cq}}$ method (25).

Small interfering (si)RNA and transfection of cell lines. A total of four siRNAs against lncRNA C22orf32-1, si-C22orf32-1, and one negative control, si-NC, were designed and synthesized by GenePharma (GenePharma, Shanghai, China). All the four si-C22orf32-1 were: Sense, 5'-CCCAGAGUCACU UAGAAGATT-3' and antisense, 5'-AUGGCCAUCAAGAUU AGGGTT-3'; sense, 5'-GAGGCGUGGAGUCUUGUUUTT-3' and antisense, 5'-AAACAAGACUCCACGCCUCTT-3'; sense, 5'-GGCGGAUUCAUUACAGUUATT-3' and antisense, 5'-UAACUGUAAUGAAUCCGCCTT-3'; sense, 5'-CUGGAA UACAAUGCUCUAUTT-3' and antisense, 5'-AUAGAGCAU UGUAUUCCAGTT-3'. A density of $1 \times 10^{5}$ NPC cells were seeded onto 6-well plates overnight and transfected with $100 \mathrm{nM}$ si-C22orf32-1 or 100 nM si-NC using Lipofectamine ${ }^{\circledR} 2000$ (Invitrogen; Thermo Fisher Scientific, Inc.). The interfering efficiency was tested using RT-qPCR $48 \mathrm{~h}$ following transfection. The most effective siRNA against si-C22orf32-1, which had a silence efficiency of $>57.3 \%$, was then selected for further analysis. The primers used were: Si-C22orf32-1 sense: 5'-CCC UAAUCUUGAUGGCCAUTT-3' and antisense: 5'-AUGGCC AUCAAGAUUAGGGTT-3'; si-NC sense: 5'-GAGGCGUGG AGUCUUGUUUTT-3' and antisense: 5'-AAACAAGACUCC ACGCCUCTT-3'.

Cell proliferation assays. The cell proliferation assay was conducted with Cell Counting Kit-8 (CCK8; Dojindo Molecular Technologies, Inc., Kumamoto, Japan). A total of $3,0006-10 \mathrm{~B}$ cells/well were seeded onto 10 replicate wells of 96-well plates. A total of 5-wells were transfected with si-NC and the others were transfected with si-C22orf32-1. Cell proliferation was monitored every $24 \mathrm{~h}$ following transfection up to $72 \mathrm{~h}$, according to the protocol of the manufacturer. All assays were performed at least three times.

Cell scratch assay. The cell scratch assay was used to detect the migration of the NPC cells. A total of $2 \times 10^{6}$ cells/well of the $6-10 \mathrm{~B}$ cell line were plated onto 6 -well plates. The cells were transfected with si-NC and si-C22orf32-1 at 90-100\% confluence. Sterile $200 \mu \mathrm{l}$ pipette tips were used to scrape a clear line through the well $6 \mathrm{~h}$ following transfection. The serum-free RPMI-1640 medium was used instead of the previous medium. The migration distance was assessed and images were captured at 0 and $24 \mathrm{~h}$ following scraping using an inverted microscope (Olympus Corporation, Tokyo, Japan). All experiments were performed in triplicate.

Transwell and matrigel assays. 6-10B cells were harvested $24 \mathrm{~h}$ following transfection with si-C22orf32-1 or si-NC. For the migration assays, $1 \times 10^{4}$ cells diluted in $100 \mu \mathrm{l}$ serum-free RPMI-1640 medium were plated into the upper chamber with a pore size $8 \mu \mathrm{m}$ (BD Biosciences, Franklin Lakes, NJ, 
USA). For the invasion assays, $1 \times 10^{4}$ cells diluted in $100 \mu 1$ serum-free RPMI-1640 medium were plated into the upper chamber, which was coated with Matrigel (BD Biosciences, San Jose, CA, USA). A total of $500 \mu 1$ medium containing $10 \%$ FBS was added to the lower chambers. Following culture for $24 \mathrm{~h}$, the cells that had invaded and migrated to the membrane were fixed with paraformaldehyde for $25 \mathrm{~min}$, then stained with $0.1 \%$ crystal violet for $25 \mathrm{~min}$. The images were captured and cells were counted using an inverted microscope (Olympus Corporation) and 5 fields of view were selected to be assessed. All experiments were performed in triplicate.

Cell apoptosis assay. 6-10B cells were plated onto 6-well plates and cultured in a humidified chamber supplemented with $5 \% \mathrm{CO}_{2}$ at $37^{\circ} \mathrm{C}$ following transfection. The cells were collected $48 \mathrm{~h}$ subsequent to this using EDTA-free pancreatic enzymes (Gibco; Thermo Fisher Scientific, Inc.) and washed three times with pre-chilled PBS. Subsequent to resuspension in $1 \mathrm{X}$ binding buffer (Invitrogen; Thermo Fisher Scientific, Inc.), the cells were stained with a propidium iodide detection kit (Invitrogen; Thermo Fisher Scientific, Inc.) and Annexin V-fluorescein isothiocyanate (Invitrogen; Thermo Fisher Scientific) in the dark for $15 \mathrm{~min}$. Flow cytometry (EPICS XI-4; Beckman Coulter, Inc., Brea, CA, USA) was used to quantify the rate of apoptosis. All experiments were performed at least three times.

Statistical analysis. All experiments were performed at least three times, therefore the data were calculated as the mean \pm standard deviation. All statistical analyses were performed using SPSS software version 17.0 (SPSS, Inc., Chicago, IL, USA). An unpaired t-test was to analyze the expression difference between NPC specimens and normal specimens, and the NPC and the normal nasopharyngeal epithelial cell lines. Student's $t$ test was used to analyze the data from the proliferation, invasion, migration, apoptosis and scratch assays. $\mathrm{P}<0.05$ (two-tailed) was considered to indicate a statistically significant difference.

\section{Results}

The expression of IncRNA C22-orf32-1 is up-regulated in NPC cell lines and human NPC clinical tissues. The expression levels of lncRNA C22orf32-1 were tested in 24 normal nasopharyngeal epithelial tissues and 24 NPC tissues. The results revealed that 1 cRNA C22orf32-1 was significantly upregulated in NPC tissues compared with normal nasopharyngeal epithelial tissues (Fig. 1A). The NPC 6-10B cell line and normal nasopharyngeal epithelial NP460 cell line were selected to verify the expression pattern of lncRNA C22orf32-1. The expression level of lncRNA C22orf32-1 in the 6-10B cell line was significantly higher compared with the NP460 cells (Fig. 1B).

Knockdown of IncRNA C22-orf32-1 inhibited the proliferation of NPC cells. To investigate the potential involvement of lncRNA C22orf32-1 in NPC progression, four siRNAs were used to decrease the expression of IncRNA C22orf32-1. The most effective siRNA was si-C22orf32-1, and further analysis
Table I. Clinicopathological characteristics of patients.

Characteristics

Number of cases

Mean age, range (years)

$43(25-64)$

Sex (male/female)

$18 / 6$

Degree of differentiation

$15 / 7$

(undifferentiated/differentiated)

Histology (squamous/others)

$24 / 0$

Lymph node metastasis (+/-)

$17 / 7$

Distal metastasis (+/-)

$0 / 24$

Clinical TNM stage (I-II/III-IV)
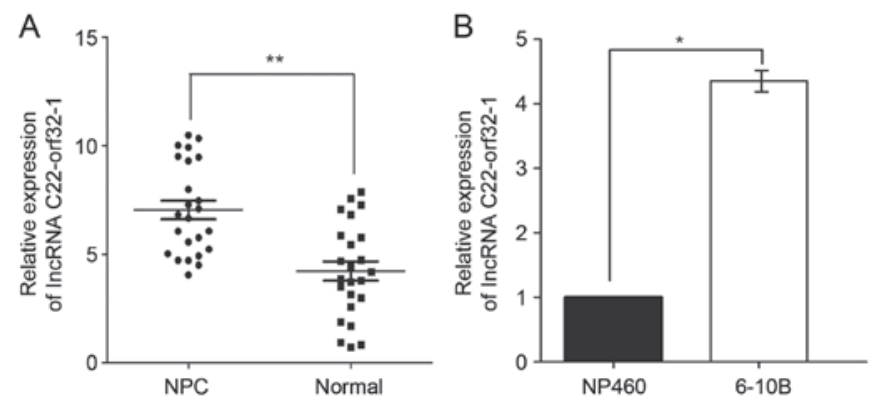

Figure 1. Relative lncRNA C22orf32-1 expression in NPC tissues and NPC cell lines assessed by quantitative reverse transcription polymerase chain reaction. (A) 1ncRNA C22orf32-1 was upregulated in 24 NPC tissues compared with 24 normal nasopharyngeal epithelial samples. (B) lncRNA C22orf32-1 presented higher expression in the NPC 6-10B cell line compared with normal nasopharyngeal epithelial NP460 cell line. The data were analyzed using $2^{-\Delta \Delta \mathrm{Cq}}$ method and presented as the mean \pm standard deviation. ${ }^{*} \mathrm{P}<0.05,{ }^{* *} \mathrm{P}<0.01$, with comparisons indicated by lines. IncRNA, long non-coding RNA; NPC, nasopharyngeal carcinoma.
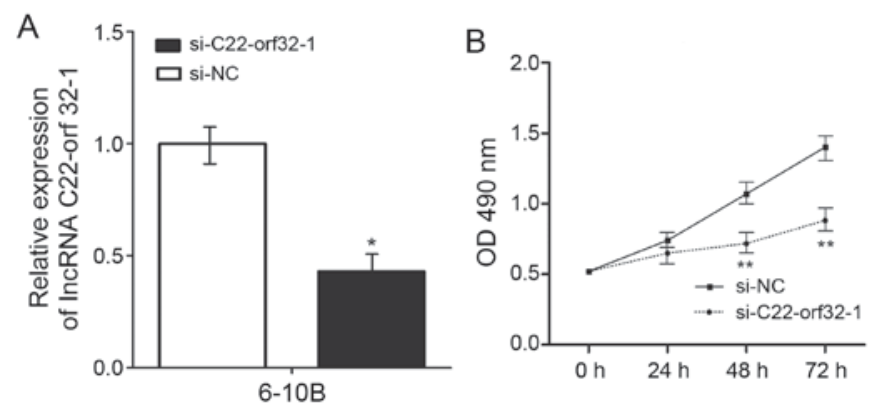

Figure 2. Knockdown of lncRNA C22orf32-1 in NPC cell lines. (A) Expression levels of 1ncRNA C22orf32-1 in 6-10B cells transfected with si-C22orf32-1 were significantly reduced compared with the cells transfected with si-NC. (B) Growth of 6-10B cells were detected by Cell Counting Kit-8 assay every $24 \mathrm{~h}$ from $0 \mathrm{~h}$ to $72 \mathrm{~h}$. IncRNA, long non-coding RNA; si, small interfering RNA; NC, negative control; OD, optical density. ${ }^{*} \mathrm{P}<0.05,{ }^{* *} \mathrm{P}<0.01$.

revealed that it significantly decreased the expression levels of lncRNA C22orf32-1 in the 6-10B cell lines by $>57.3 \%$ $(\mathrm{P}<0.05$; Fig. 2A). CCK-8 assays were used to examine the effect of 1ncRNA C22orf32-1 on the proliferation of NPC cells. Knockdown of lncRNA C22orf32-1 significantly suppressed the growth of the 6-10B cell line 48 and $72 \mathrm{~h}$ following transfection compared with the negative control (Fig. 2B). 


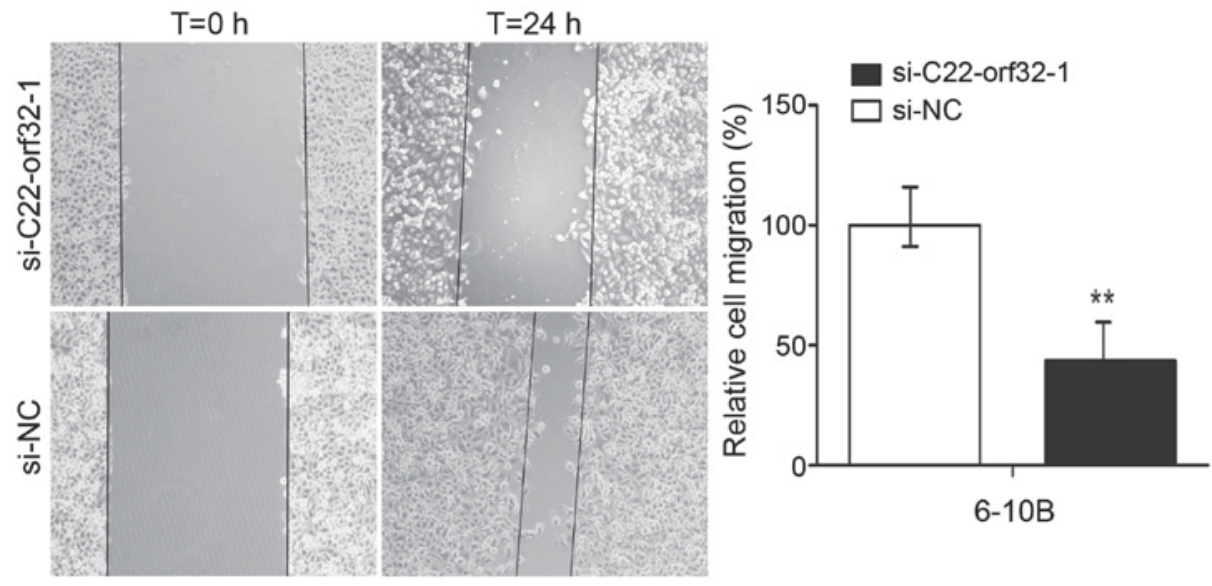

Figure 3. Long non-coding RNA C22orf32-1 promoted the migration of nasopharyngeal carcinoma cells. Cell scratch assays were used to detect the migration of the 6-10B cell line following transfection with si-C22orf32-1 and si-NC. The spacing measured from the cell transfected si-C22orf32-1 was obviously wider than the cell transfected si-NC. Magnification, x100. si, small interfering RNA; NC, negative control; T, time. " $\mathrm{P}<0.01$.
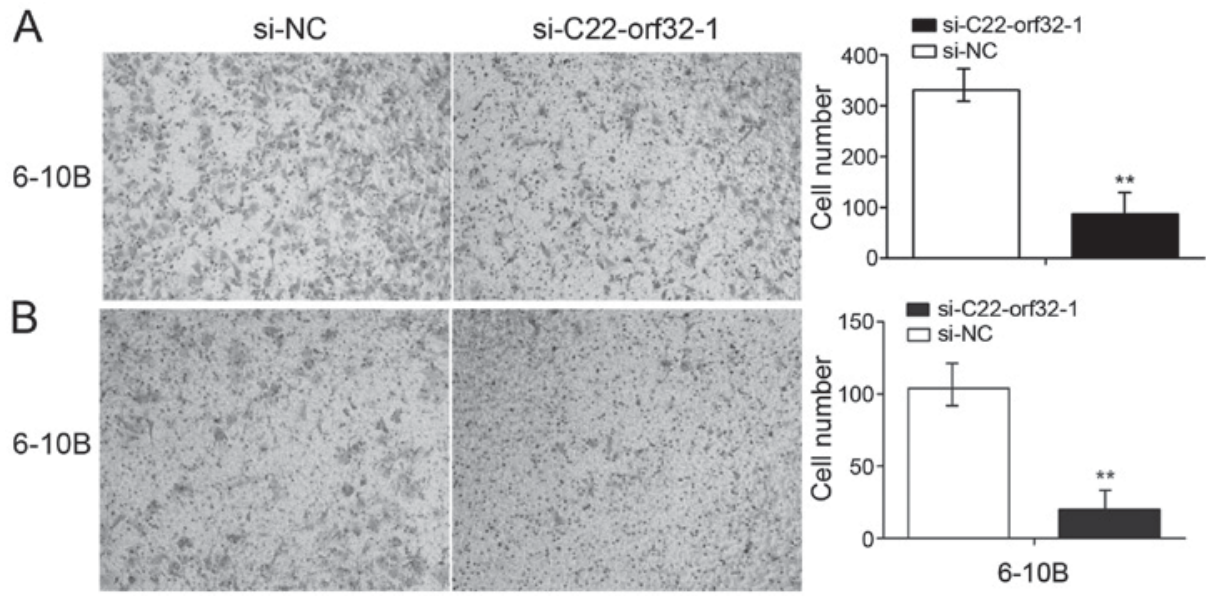

Figure 4. Inhibition of long non-coding RNA C22orf32-1 suppresses the invasion and migratory capacity of NPC cells. (A) Transwell migration assays and (B) Matrigel invasion assays demonstrated the migration and invasion activities of the 6-10B cell line subsequent to transfection with si-C22orf32-1 and si-NC. si, small interfering RNA; NC, negative control. ${ }^{* *} \mathrm{P}<0.01,{ }^{* * *} \mathrm{P}<0.001$.
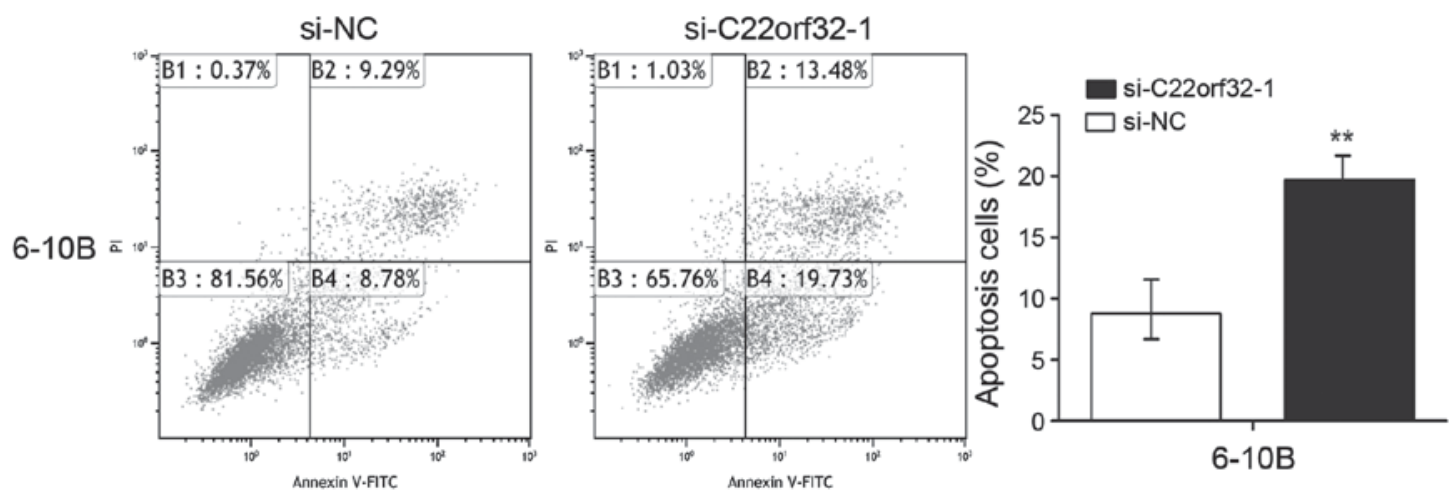

Figure 5. Long non-coding RNA C22orf32-1 decreased apoptosis of the NPC cells. Flow cytometry was used to analyze the apoptosis rates of the NPC 6-10B cell line following transfection with si-C22orf32-1 and si-NC. PI, propidium iodide; si, small interfering RNA; NC, negative control; NPC, nasopharyngeal carcinoma; FITC, fluorescein isothiocyanate. ${ }^{* *} \mathrm{P}<0.01,{ }^{* * * *} \mathrm{P}<0.001$

LncRNA C22orf32-1 promoted NPC cell migration and invasion. The scratch assays and Transwell migration assays were used to detect the effect of IncRNA C22orf32-1 on the migratory ability of NPC cells. In the scratch assays, it was revealed that the migration abilities of the cells transfected with si-C22orf32-1 were significantly reduced compared with the cells transfected with si-NC. The migration rates were reduced to $58 \%$ in $6-10 \mathrm{~B}$ cells $(\mathrm{P}<0.001$; Fig. 3). Similarly, 
the migration rates of the 6-10B cell lines following transfection with si-C22orf32-1 were decreased by $64.3 \%(\mathrm{P}<0.001$; Fig. 4A) compared with the negative control. The Matrigel invasion assays were performed to investigate whether lncRNA C22orf32-1 was involved in the invasion of NPC cells. The results revealed that lncRNA C22orf32-1 knockdown cells exhibited impeded invasion abilities in the 6-10B cell line, and were reduced by $76.2 \%$ ( $\mathrm{P}<0.001$; Fig. 4B).

Knockdown of IncRNA C22orf32-1 promoted NPC cell apoptosis. To investigate whether lncRNA C22orf32-1 was involved in the progression of NPC cell apoptosis, flow cytometry was used to analysis the rates of apoptosis in the 6-10B cells. The flow cytometry assay results indicated that the levels of apoptosis in the cells transfected with si-C22orf32-1 were significantly increased compared with the cells transfected with si-NC, with 8.78 vs. $19.73 \%$ $(\mathrm{P}<0.001$; Fig. 5).

\section{Discussion}

Epidemiological studies have demonstrated that NPC is a specific regional genetic disease with multiple factors. Exposure to the Epstein-Barr virus, the environment and genetics are the three main factors that influence the development of NPC (23,26-28). Like multiple other human malignancies, the occurrence of NPC is due to the activation of oncogenes and/or the inactivation of tumor suppressor genes that disrupt the homeostasis of cellular gene expression.

LncRNA is considered an important factor that influences the development of human tumors $(29,30)$. Previous studies have demonstrated that abnormal expression of lncRNAs was able to change the biological functions of tumor cells by affecting various cellular processes. According to their alternative expression pattern, certain IncRNAs may reflect the progress of disease, and may be regarded as independent predictors for cancer diagnosis $(14,16,31,32)$. The overexpression of Hox transcript antisense RNA in breast cancer promoted metastasis $(33,34)$ and homeobox A transcript at the distal tip is considered an indicator for determining the progress of pancreatic cancer (35). Prostate cancer associated transcript 1 identified in hepatocellular carcinoma has been associated with the prognosis in patients with hepatocellular carcinoma (36). Yet, the specific functions of lncRNA in the occurrence of tumors remains unclear.

As demonstrated in a previous study, the expression of 1ncRNA C22orf32-1 was upregulated in primary NPC tissues (22). However, the mechanism of lncRNA C22orf32-1 in the development of NPC remains unknown (22). In the present study, the expression of lncRNA C22orf32-1 was examined in 24 primary NPC tissues and 24 normal nasopharyngeal epithelial tissues, and in one NPC and one normal nasopharyngeal epithelial cell line. It was revealed that the expression levels of lncRNA C22orf32-1 were higher in the NPC tissues and the NPC cell line. IncRNA C22orf32-1 is located in the human chromosome 22 with a length of $545 \mathrm{bp}$. Genetic diversity is a feature of chromosome 22, and as such this chromosome is associated with the occurrence of human disease. At present, at least 27 types of disease are associated with chromosome 22, particularly malignancies including acute lymphoid leukemia, chronic myelogenous leukemia and malignant rhabdoid tumors (37-39). The high expression level and the specific location of lncRNA C22orf32-1 indicate that it may be associated with NPC. The effect of 1ncRNA C22orf32-1 knockdown on NPC cells was investigated, and it was demonstrated that the overexpression of lncRNA C22orf32-1 in the NPC cell line promoted cell proliferation, migration, invasion, and suppressed cell apoptosis. Conversely, subsequent to lncRNA C22orf32-1 knockdown, the capacities for proliferation, migration and invasion were decreased, and apoptosis was increased. This indicated that 1ncRNA C22orf32-1 is a cancer-associated gene involved in NPC, which may be a useful candidate biomarker for the early detection and treatment of NPC. Additional studies are required to determine the potential mechanisms of lncRNA C22orf32-1 in the development of NPC.

\section{Acknowledgements}

The present study was supported by the Science and Technology Development Fund Project of Shenzhen (grant nos. JCYJ20120827150357364, JCYJ20130402114702127 and JCYJ2015040309144336) and the Medical Research Project of the Health and Family Planning Commission of Shenzhen (grant no. 201302005).

\section{References}

1. Agulnik M and Epstein JB: Nasopharyngeal carcinoma: Current management, future directions and dental implications. Oral Oncol 44: 617-627, 2008.

2. Lee AW, Lin JC and Ng WT: Current management of nasopharyngeal cancer. Semin Radiat Oncol 22: 233-244, 2012.

3. Spano JP, Busson P, Atlan D, Bourhis J, Pignon JP, Esteban C and Armand JP: Nasopharyngeal carcinomas: An update. Eur J Cancer 39: 2121-2135, 2003.

4. Vokes EE,Liebowitz DN and Weichselbaum RR: Nasopharyngeal carcinoma. Lancet 350: 1087-1091, 1997.

5. August M, Dodson TB, Nastri A and Chuang SK: Nasopharyngeal carcinoma: Clinical assessment and review of 176 cases. Oral Surg Oral Med Oral Pathol Oral Radiol Endod 91: 205-214, 2001.

6. Chen W and Hu GH: Biomarkers for enhancing the radiosensitivity of nasopharyngeal carcinoma. Cancer Biol Med 12: 23-32, 2015.

7. Wolff HA, Rödel RM, Gunawan B, Overbeck T, Herrmann MK, Hennies S, Hille A, Vorwerk H, Matthias C, Hess CF and Christiansen H: Nasopharyngeal carcinoma in adults: Treatment results after long-term follow-up with special reference to adjuvant interferon-beta in undifferentiated carcinomas. J Cancer Res Clin Oncol 136: 89-97, 2010.

8. Chou J, Lin YC, Kim J, You L, Xu Z, He B and Jablons DM: Nasopharyngeal carcinoma-review of the molecular mechanisms of tumorigenesis. Head Neck 30: 946-963, 2008.

9. Chen ZT, Liang ZG and Zhu XD: A Review: Proteomics in nasopharyngeal carcinoma. Int J Mol Sci 16: 15497-15530, 2015.

10. Razak AR, Siu LL, Liu FF, Ito E, O'Sullivan B and Chan K: Nasopharyngeal carcinoma: The next challenges. Eur J Cancer 46: 1967-1978, 2010.

11. Tulalamba W and Janvilisri T: Nasopharyngeal carcinoma signaling pathway: An update on molecular biomarkers. Int $\mathbf{J}$ Cell Biol 2012: 594681, 2012.

12. Morris KV and Mattick JS: The rise of regulatory RNA. Nat Rev Genet 15: 423-437, 2014.

13. Yarmishyn AA and Kurochkin IV: Long noncoding RNAs: A potential novel class of cancer biomarkers. Front Genet 6: 145, 2015.

14. Ulitsky I and Bartel DP: lincRNAs: Genomics, evolution, and mechanisms. Cell 154: 26-46, 2013. 
15. Saxena A and Carninci P: Long non-coding RNA modifies chromatin: Epigenetic silencing by long non-coding RNAs. Bioessays 33: 830-839, 2011.

16. Zhang W, Wang L, Zheng F, Zou R, Xie C, Guo Q, Hu Q, Chen J, Yang X, Yao H, et al: Long noncoding RNA expression signatures of metastatic nasopharyngeal carcinoma and their prognostic value. Biomed Res Int 2015: 618924, 2015.

17. Gibb EA, Brown CJ and Lam WL: The functional role of long non-coding RNA in human carcinomas. Mol Cancer 10: 38, 2011.

18. Maruyama R and Suzuli H: Long noncoding RNA involvement in cancer. BMB Rep 45: 604-611, 2012.

19. Xu T, Su B, Wang C, Wang S, Huang H, Pan Y, Wang D, Wei W, Claret FX and Yang H: Molecular markers to assess short-term disease local recurrence in nasopharyngeal carcinoma. Oncol Rep 33: 1418-1426, 2015.

20. Su YJ, Yu J, Huang YQ and Yang J: Circulating long noncoding RNA as a potential target for prostate cancer. Int $\mathrm{J}$ Mol Sci 16 : 13322-13338, 2015.

21. Weber DG, Johnen G, Casjens S, Bryk O, Pesch B, Jöckel KH, Kollmeier $\mathrm{J}$ and Brüning T: Evaluation of long noncoding RNA MALAT1 as a candidate blood-based biomarker for the diagnosis of non-small cell lung cancer. BMC Res Notes 6: 518, 2013.

22. Gao W, Chan JY and Wong TS: Differential expression of long noncoding RNA in primary and recurrent nasopharyngeal carcinoma. Biomed Res Int 2014: 404567, 2014.

23. Zhou X, Cui J, Macias V, Kajdacsy-Balla AA, Ye H, Wang J and Rao PN: The progress on genetic analysis of nasopharyngeal carcinoma. Comp Funct Genomics 2007: 57513, 2007.

24. Edge SB and Compton CC: The America joint committee on cancer: The 7th edition of the AJCC cancer staging manual and the future of TNM. Ann Surg Oncol 17: 1471-1474, 2010.

25. Livak KJ and Schmittgen TD: Analysis of relative gene expression data using real-time quantitative PCR and the 2(-Delta Delta C(T)) Method. Methods 25: 402-408, 2001.

26. Chang ET and Adami HO: The enigmatic epidemiology of nasopharyngeal carcinoma. Cancer Epidemiol Biomarkers Prev 15. 1765-1777, 2006
27. Kimura Y, Suzuki D, Tokunaga T, Takabayashi T, Yamada T, Wakisaka N, Yoshizaki T, Murata H, Miwa K, Shoujaku H, et al: Epidemiological analysis of nasopharyngeal carcinoma in the central region of Japan during the period from 1996 to 2005 . Auris Nasus Larynx 38: 244-249, 2011.

28. Young LS and Dawson CW: Epstein-Barr virus and nasopharyngeal carcinoma. Chin J Cancer 33: 581-590, 2014.

29. Wapinski $\mathrm{O}$ and Chang HY: Long noncoding RNAs and human disease. Trends Cell Biol 21: 354-361, 2011.

30. Qiu MT, Hu JW, Yin R and Xu L: Long noncoding RNA: An emerging paradigm of cancer research. Tumour Biol 34: 613-620, 2013.

31. Quan M, Chen J and Zhang D: Exploring the secrets of long noncoding RNAs. Int J Mol Sci 16: 5467-5496, 2015.

32. Rinn JL and Chang HY: Genome regulation by long noncoding RNAs. Annu Rev Biochem 81: 145-166, 2012.

33. Yao Y, Li $\mathbf{J}$ and Wang L: Large intervening non-coding RNA HOTAIR is an indicator of poor prognosis and a therapeutic target in human cancers. Int J Mol Sci 15: 18985-18999, 2014.

34. Zhang J, Zhang P, Wang L, Piao HL and Ma L: Long non-coding RNA HOTAIR in carcinogenesis and metastasis. Acta Biochim Biophys Sin (Shanghai) 46: 1-5, 2014.

35. Li Z, Zhao X, Zhou Y, Liu Y, Zhou Q, Ye H, Wang Y, Zeng J, Song Y, Gao W, et al: The long non-coding RNA HOTTIP promotes progression and gemcitabine resistance by regulating HOXA13 in pancreatic cancer. J Transl Med 13: 84, 2015.

36. Yan TH, Yang H, Jiang JH, Lu SW, Peng CX, Que HX, Lu WL and Mao JF: Prognostic significance of long non-coding RNA PCAT-1 expression in human hepatocellular carcinoma. Int J Clin Exp Pathol 8: 4126-4131, 2015.

37. De Decker HP and Lawrenson JB: The 22q11.2 deletion: From diversity to a single gene theory. Genet Med 3: 2-5, 2001.

38. Brinchi V, Curatolo P, Di Franco C and Dallapiccola B: 2 new cases of chromosome 22 with ring structure. Pathologica 71: 369 , 1979 (In Italian).

39. Schneider M and Eliez S: 22q11.2 microdeletion. Arch Pediatr 17: 431-434, 2010 (In French). 